Revista de Metalurgia 52(1)

Enero-Marzo 2016, e062

ISSN-L: 0034-8570

doi: http://dx.doi.org/10.3989/revmetalm.062

\title{
Elución de iones de níquel desde esferas de alginato en un medio ácido
}

\author{
Alvaro Aracena ${ }^{\mathrm{a}, \bowtie}$, Francisco Cárcamo $^{\mathrm{a}}$, Óscar Jerez ${ }^{\mathrm{b}}$, Robinson Constanzo ${ }^{\mathrm{a}}$ \\ ${ }^{\text {a}}$ Pontificia Universidad Católica de Valparaíso, Escuela de Ingeniería Química, General Cruz 34, Valparaíso, Chile \\ ${ }^{b}$ Universidad de Concepción, Instituto de Geología Económica Aplicada (GEA), Casilla 160-C, Concepción, Chile \\ ${ }^{\otimes}$ Autor para la correspondencia: alvaro.aracena@pucv.cl
}

Enviado: 20 Abril 2015; Aceptado: 13 Diciembre 2015; Publicado on-Line: 17 Febrero 2016

RESUMEN: Se estudió la elución de iones de níquel desde esferas de alginato en un intervalo de temperaturas de 2 a $80^{\circ} \mathrm{C}$ y una concentración de ácido sulfúrico entre 0,005 a 2,5 g L ${ }^{-1}$. El mecanismo de elución fue establecido por intercambio iónico entre protones e iones de níquel alcanzando valores de elución de $93 \%$ a una temperatura de $80{ }^{\circ} \mathrm{C}$ y concentración de $\mathrm{H}_{2} \mathrm{SO}_{4}$ de $0,25 \mathrm{~g} \mathrm{~L}^{-1}$. También se estudió la influencia de otros ácidos sobre la elución de níquel. La velocidad de elución de níquel fue significativamente influenciada por la temperatura y concentración de $\mathrm{H}_{2} \mathrm{SO}_{4}$. La cinética de elución de iones de níquel fue estudiada y el modelo $1-(1-\alpha)^{1 / 3}=k_{a p p} \mathrm{t}$ describió muy bien la cinética de esta reacción. La dependencia de la elución de níquel sobre la concentración de ácido sulfúrico fue del orden de 0,33 . Además, fueron determinadas las constantes de velocidad intrínseca y se obtuvo un valor de energía de activación de $54,5 \mathrm{~kJ} \mathrm{~mol}^{-1}$ para el intervalo de temperaturas estudiado. Los resultados indicaron que el proceso de elución de níquel fue controlado por reacciones químicas.

PALABRAS CLAVE: Cinética intrínseca; Esferas de alginato; Iones de níquel; Velocidad de elución

Citation / Cómo citar este artículo: Aracena, A., Cárcamo, F., Jerez, Ó., Constanzo, R. (2016) "Elución de iones de níquel desde esferas de alginato en un medio ácido". Rev. Metal. 52(1):e062. doi: http://dx.doi.org/10.3989/revmetalm.062.

ABSTRACT: Elution of nickel ions from alginate beads in an acid media. Elution of nickel ions from alginate beads was studied in a temperature range of 2 to $80{ }^{\circ} \mathrm{C}$ and a sulfuric acid concentration between 0.005 to $2.5 \mathrm{~g} \mathrm{~L}^{-1}$. The elution mechanism was established by ion exchange between nickel and ions protons, obtaining a value of $93 \%$ elution at a temperature of $80^{\circ} \mathrm{C}$ and $\mathrm{a} \mathrm{H}_{2} \mathrm{SO}_{4}$ concentration of $0.25 \mathrm{~g} \mathrm{~L}^{-1}$. The influence of other acids on nickel elution was also studied. The nickel elution rate was significantly influenced by temperature and concentration of $\mathrm{H}_{2} \mathrm{SO}_{4}$. Elution kinetics of nickel ions was studied, and the 1-(1- $\left.\alpha\right)^{1 / 3}=k_{a p p}$ t model properly described the kinetics of this reaction. The dependence of nickel elution on the sulfuric acid concentration was of the order of 0.33 . Moreover, the intrinsic rate constants were determined and an activation energy value of $54.5 \mathrm{~kJ} \mathrm{~mol}^{-1}$ was obtained for the temperature range studied. The results indicated that the nickel elution is a process controlled by chemical reactions.

KEYWORDS: Alginate beads; Elution rate; Intrinsic kinetics; Nickel ions

Copyright: (C) 2016 CSIC. This is an open-access article distributed under the terms of the Creative Commons Attribution-Non Commercial (by-nc) Spain 3.0 License. 


\section{INTRODUCCIÓN}

Industrias involucradas en la metalurgia, eléctrica y electrónica, minería y fundición, industria del chapado de metales, entre otros, liberan metales pesados hacia las aguas residuales, causando un daño al ecosistema (Xia et al., 2014). Tales aguas residuales necesitan ser tratadas para disminuir el impacto ambiental. El níquel es uno de los metales liberados $\mathrm{y}$, al igual que con otros metales de transición, es muy tóxico para los humanos y otros organismos (Anoop et al., 2011; Belova et al., 2014). Consecuentemente, es necesario eliminar estos metales para asegurar adecuadamente un efluente de calidad para varios usos (Malanis y Katsou, 2013), ya que además pueden reducir la eficiencia de tratamientos biológicos (Vocciante et al., 2014). Existen algunos procesos para la extracción de metales pesados desde aguas residuales que incluyen precipitación química, adsorción, intercambio iónico, flotación, filtración por membrana, tratamiento electroquímico y coagulaciónfloculación (O’Connell et al., 2008). Los procesos de adsorción e intercambio iónico pueden ser utilizados para la extracción de metales pesados tal como los iones de níquel. La ventaja competitiva de tales procesos aumenta cuando se utilizan por el bajo costo de tratamiento. Los materiales poliméricos naturales tales como esferas de alginato, que es un sorbente que tiene tamaño controlado, buenas propiedades físicas y químicas y alta porosidad, han mostrado su utilidad para la extracción de metales pesados tales como cadmio (Ibáñez y Umetsu, 2008), zinc (Ibáñez y Aracena, 2014), níquel (Aracena et al., 2015), entre otros.

Aracena et al. (2015) demostró que utilizando las esferas protonadas secas de alginato (PDAB en inglés) se puede extraer $\mathrm{Ni}^{2+}$ desde soluciones muy diluidas. Se alcanzó un valor de extracción de iones de níquel de $75,9 \mathrm{mg} \mathrm{g}^{-1}$ de alginato seco a $\mathrm{pH}=5,0 \mathrm{y}$ un tiempo de 360 minutos. El mecanismo de extracción fue por intercambio iónico entre los iones de níquel y protones provenientes de los grupos funcionales de las esferas de alginato. Lamentablemente, no se realizaron estudios sobre la elución de níquel para conocer los parámetros que afectan en la desorción de este elemento desde el PDAB. Hay que destacar además que no existen estudios bibliográficos sobre elución de iones de níquel.

Este trabajo de investigación tiene como objetivo estudiar los mecanismos de elución de iones de níquel desde las esferas de alginato bajo condiciones experimentales tales como agitación, temperatura, concentración de ácido sulfúrico y diferentes ácidos.

\subsection{Química y mecanismo de elución de $\mathbf{N i}^{2+}$}

Se realizó un estudio termodinámico para conocer la solubilidad de los constituyentes del níquel. Los diagramas de fases electroquímicos (diagramas Eh-pH) son muy utilizados para predecir las

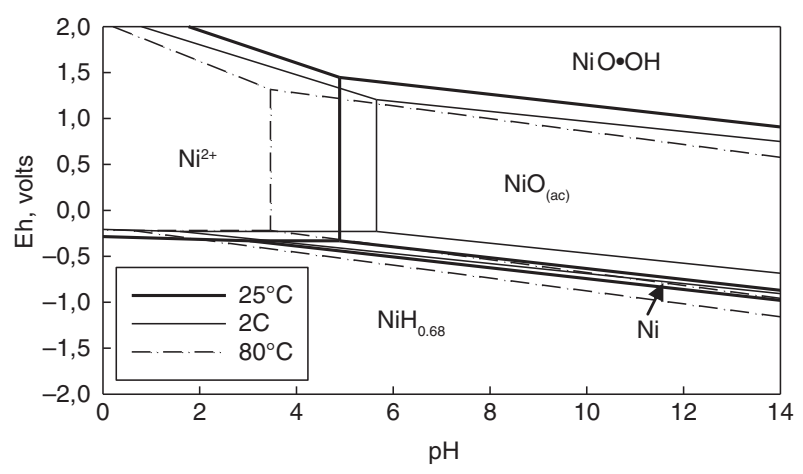

FigurA 1. Diagrama Eh-pH del sistema $\mathrm{Ni}-\mathrm{H}_{2} \mathrm{O}$ a una concentración de $\mathrm{Ni}=2,9 \times 10^{-4} \mathrm{~mol} \mathrm{~L}^{-1}$. Líneas finas: equilibrios obtenidos a $2{ }^{\circ} \mathrm{C}$. Líneas gruesas: equilibrios obtenidos a $25^{\circ} \mathrm{C}$. Líneas Entrecortadas: equilibrios obtenidos a $55^{\circ} \mathrm{C}$.

condiciones de estabilidad o inestabilidad de minerales en contacto con soluciones acuosas. Por esta razón se construyó el diagrama Eh-pH para el sistema $\mathrm{Ni}-\mathrm{H}_{2} \mathrm{O}$ a una temperatura de 2,25 y $80{ }^{\circ} \mathrm{C}$ considerando la concentración de níquel de $0,00029 \mathrm{~mol} \mathrm{~L}^{-1}$. Los datos termodinámicos utilizados en la construcción de este diagrama en equilibrio fueron obtenidos principalmente desde la base de datos del programa HSC Chemistry (Roine, 1999). Los componentes de níquel presentes en este diagrama fueron el $\mathrm{Ni}^{2+}, \mathrm{NiO}, \mathrm{NiH}_{0.18}$ y NiO·OH. La Fig. 1 muestra el diagrama de equilibrio del sistema $\mathrm{Ni}-\mathrm{H}_{2} \mathrm{O}$ a 2 , 25 y $80{ }^{\circ} \mathrm{C}$. En este diagrama se observa que el área de predominancia del $\mathrm{Ni}^{2+}$, ión fundamental en el intercambio iónico, está sobre los valores de potencial de $-0,25$ volts y valores de $\mathrm{pH}$ ácidos $(\mathrm{pH}<6,0)$. A medida que se incrementaba la temperatura, el área de predominancia del catión níquel se volvió más amplio. Así, para una temperatura de 2 y $80^{\circ} \mathrm{C}$, las líneas de equilibrio entre el $\mathrm{Ni}^{2+}$ y $\mathrm{NiO}_{(\mathrm{ac})}$ se encuentra a valores de $\mathrm{pH}$ de 3,5 y 5,7, respectivamente. En cambio, los rangos de Eh (línea de equilibrio entre $\mathrm{Ni}^{2+}$ y $\mathrm{NiH}_{0.68}$ ) se mantienen con pequeñas diferencias a pesar del incremento de la temperatura.

En el rango de $\mathrm{pH}$ estudiado, en donde predomina el ión níquel $\left(\mathrm{Ni}^{2+}\right)$, se puede concluir que es posible que se presente un mecanismo de intercambio iónico el cual sería viable desde una temperatura entre 2 a $80{ }^{\circ} \mathrm{C}$ para un rango de $\mathrm{pH}$ ácido. Por lo tanto, este intercambio iónico puede ser representado por la reacción (1):

$\{\mathrm{COO}\}_{2}-\mathrm{Ni}+2 \mathrm{H}^{+}=2\{\mathrm{COO}-\mathrm{H}\}+\mathrm{Ni}^{2+}$

\section{MATERIALES Y MÉTODOS}

Todos los agentes utilizados fueron de alta pureza y no se necesitó de un tratamiento adicional. Para los trabajos desarrollados de extracción y elución, los análisis químicos fueron hechos por espectroscopía 
de absorción atómica (EAA). Las soluciones con diferentes concentraciones de níquel fueron preparadas disolviendo $\mathrm{NiSO}_{4} * 6 \mathrm{H}_{2} \mathrm{O}$ en agua doblemente destilada. Para el análisis de la composición química y morfológica de las esferas de alginato se utilizó un microscopio electrónico de barrido (SEM) y un detector de energía dispersiva (EDS). Para la observación y análisis de las muestras de esferas de alginatos, éstas fueron cortadas con oro y grafito, respectivamente. Las esferas protonadas secas de alginato (PDAB) fueron generadas de acuerdo al protocolo descrito previamente (Ibáñez y Umetsu, 2002). Las cadenas de ácido algínico son entrecruzados con calcio y bario para generar PDAB-Ca y PDAB-Ba, que han mostrado propiedades químicas y físicas apropiadas en soluciones acuosas (Ibáñez y Umetsu, 2002). La formación del PDAB produce una superficie corrugada con un diámetro de esfera de alrededor de $1,0 \pm 0,1 \mathrm{~mm}$ el cual tiene una alta porosidad. Las esferas protonadas secas de alginato son estables en un alto rango de $\mathrm{pH}$ para valores de aproximadamente entre 1 y 6 , después de este valor el calcio y bario son liberados desde las esferas, en donde el alginato pierde rigidez y estabilidad.

\subsection{Trabajo de extracción de iones de níquel}

Los experimentos de extracción se realizaron poniendo en contacto $400 \mathrm{~mL}$ de una solución de níquel a una concentración de $17 \mathrm{mg} \mathrm{L}^{-1}$ con $80 \mathrm{mg}$ de PDAB durante un tiempo de 360 minutos a $25,0 \pm 0,1^{\circ} \mathrm{C}$. El pH de la solución se mantuvo constante por la adición de solución de $\mathrm{NaOH}$. En cada experimento se extrajo $1 \mathrm{~mL}$ de muestra a diferentes intervalos de tiempo para ser enviadas a EAA. Las muestras eran diluidas a $10 \mathrm{~mL}$ con agua doblemente destilada y luego filtradas a través de filtros de membranas de celulosa. Las concentraciones remanentes de iones de níquel, bario y calcio en los filtrados eran analizadas por EAA. Las esferas eran recopiladas al final de los experimentos, en donde fueron lavadas consecutivamente con agua doblemente destilada, secadas con aire y almacenadas para observaciones por SEM-EDS.

\subsection{Elución de iones de níquel}

Los experimentos de elución isotérmicos de tipo Batch fueron realizados en un reactor de vidrio de $2 \mathrm{~L}$ equipado con un agitador mecánico variable, una manta calefactora, un termopar, un tubo poroso para muestras líquidas y un condensador enfriado por agua para minimizar las pérdidas de solución por evaporación. El volumen de solución utilizado fue de $400 \mathrm{~mL}$. La concentración de ácido sulfúrico fue variable (desde 0,005 a $2,5 \mathrm{~g} \mathrm{~L}^{-1}$ ). $80 \mathrm{mg}$ de las esferas de alginato cargadas con níquel se pusieron en contacto con la solución de ácido sulfúrico a una temperatura variable (desde 2 a $80^{\circ} \mathrm{C}$ ).
Se extrajeron muestras de $1 \mathrm{~mL}$ cada cierto tiempo y se diluyeron a $10 \mathrm{~mL}$ con agua doblemente destilada. Las concentraciones de Ni, Ba y $\mathrm{Ca}$ se analizaron por EAA. Las esferas se recuperaron, lavaron, secaron y se analizaron mediante SEM-EDS.

Los experimentos de extracción y elución se realizaron por duplicado y triplicado, respectivamente. Las variaciones en los resultados fue, en todos los casos, inferior al $2 \%$. Los valores presentados en este trabajo, son por tanto, valores promedio.

\section{RESULTADOS Y DISCUSIÓN}

Algunas de las principales variables estudiadas que afectaron a la cinética de elución de níquel desde el PDAB fueron: velocidad de agitación, temperatura, concentración de ácido sulfúrico y diferentes ácidos. Pero primero se mostrarán algunos resultados de extracción de níquel como también análisis del alginato.

\subsection{Prueba de carga del PDAB (extracción)}

Se realizaron pruebas de cargas del alginato con iones de níquel. Se utilizó una concentración inicial de $\mathrm{Ni}=17 \mathrm{mg} \mathrm{L}^{-1}$, un valor de $\mathrm{pH}$ de 5,0 , una masa de alginato de $80 \mathrm{mg}$ y una temperatura de $25^{\circ} \mathrm{C}$. Los resultados se muestran en la Fig. 2 como concentración de níquel en función del tiempo experimental. En esta figura se puede observar un importante decrecimiento de la concentración de níquel en solución en función del tiempo experimental, en donde no se asocia la liberación de bario o calcio. Tal comportamiento ya fue demostrado y explicado en un trabajo realizado por Aracena et al. (2015). En resumen, el proceso de extracción se debe a un mecanismo de intercambio iónico en el que se intercambian los protones de los grupos funcionales del PDAB e iones de níquel de la solución.

La extracción de iones de níquel alcanzó un valor de alrededor de $77 \mathrm{mg} \mathrm{g}^{-1}$ de PDAB (peso seco)

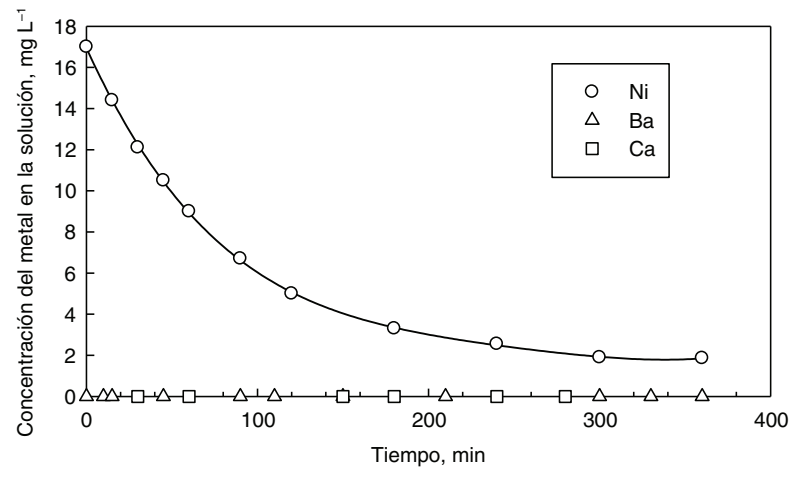

FIGURA 2. Datos de extracción típicos de concentración residual de Ni en la solución. Condiciones de trabajo de concentración inicial de $\mathrm{Ni}=17,0 \mathrm{mg} \mathrm{L}^{-1}, \mathrm{pH}=5.0$ y $\mathrm{PDAB}=80 \mathrm{mg}$. 
después de 360 minutos de tiempo de contacto. Por lo tanto, y en base a estos resultados, cada vez que se realizaba un experimento de elución, se debía pasar por este paso de carga del alginato con las mismas condiciones experimentales.

Para evidenciar la cantidad de níquel que se encontraba intercambiado en el alginato, se analizó la composición de este elemento a través de las esferas de alginato usando un análisis por topografía de superficie (BEI) con cuantificación por EDS. El perfil de concentración de níquel fue a través de la partícula de alginato y fue medida a lo largo de la línea punteada presentada en la Fig. 3. Los valores de las mediciones están dados en la Tabla 1 como porcentaje de níquel en cada punto analizado. Estos resultados indicaron que la concentración de níquel a través de la esfera de alginato es alta en la superficie externa. Este comportamiento es consistente con una alta extracción de níquel desde las

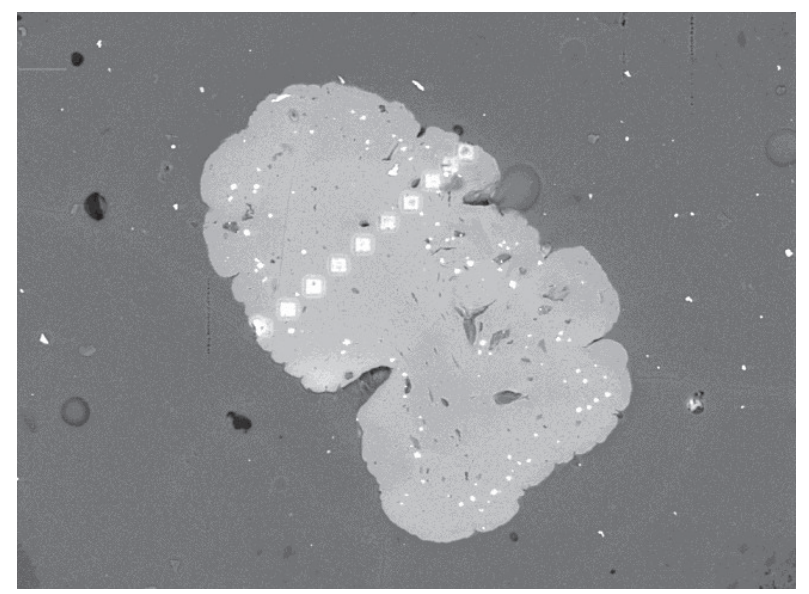

FIGURA 3. Análisis por topografía de superficie (BEI) con cuantificación por EDS de una esfera cortada de alginato para una muestra tratada. La concentración de Ni fue medida a lo largo de la línea punteada.

TABla 1. Perfil de concentración de $\mathrm{Ni}$ a través del alginato por EDS

\begin{tabular}{lc}
\hline & Concentración de Ni (\%) \\
\cline { 2 - 2 } Punto & $\mathbf{p H = 2 , 5}$ \\
\hline 1 & 2,37 \\
2 & 3,17 \\
3 & 3,98 \\
4 & 4,61 \\
5 & 5,23 \\
6 & 4,82 \\
7 & 4,62 \\
8 & 3,55 \\
9 & 2,95 \\
10 & 2,55 \\
\hline
\end{tabular}

soluciones. Por lo tanto, esta cantidad de níquel en el alginato evidencia la extracción de iones de níquel desde soluciones diluidas con esferas de alginato.

\subsection{Efecto de la velocidad de agitación}

Los experimentos de elución se llevaron a cabo entre un rango de 0 a $400 \mathrm{rpm}$ con $0,4 \mathrm{~L}$ de solución donde su concentración de $\mathrm{H}_{2} \mathrm{SO}_{4}$ fue de $0,25 \mathrm{~g} \mathrm{~L}^{-1}$ con una masa de alginato de $80 \mathrm{mg}$ y una temperatura de $25^{\circ} \mathrm{C}$. Esta solución acuosa fue la solución estándar de elución que se utilizó posteriormente. Los resultados obtenidos son presentados en la Fig. 4, los cuales mostraron que la velocidad de agitación no tuvo un efecto significativo sobre la velocidad de disolución de níquel para valores superiores a $300 \mathrm{rpm}$. Asimismo se utilizó una velocidad de $400 \mathrm{rpm}$ para el resto de experimentos para asegurar que la reacción de elución no sea controlada por la transferencia de masa.

\subsection{Efecto de la temperatura sobre la velocidad de elución}

Los experimentos fueron llevados a cabo en un intervalo de temperatura de 2 a $80{ }^{\circ} \mathrm{C}$. Los resultados son mostrados en la Fig. 5 donde se observa

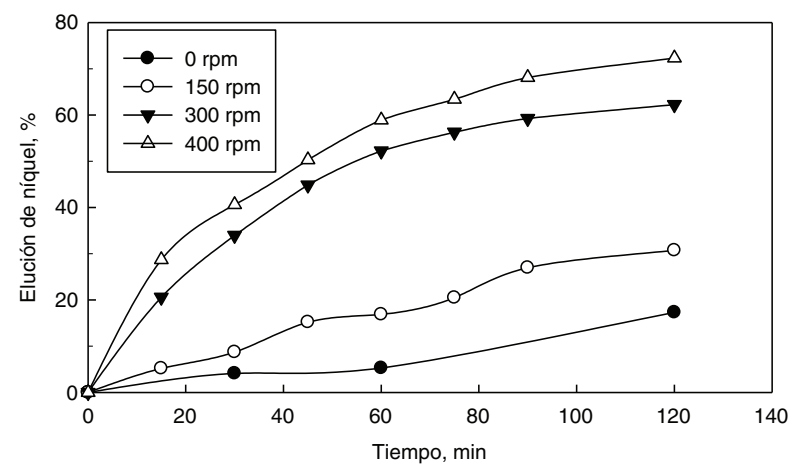

Figura 4. Efecto de la velocidad de agitación sobre la elución de níquel.

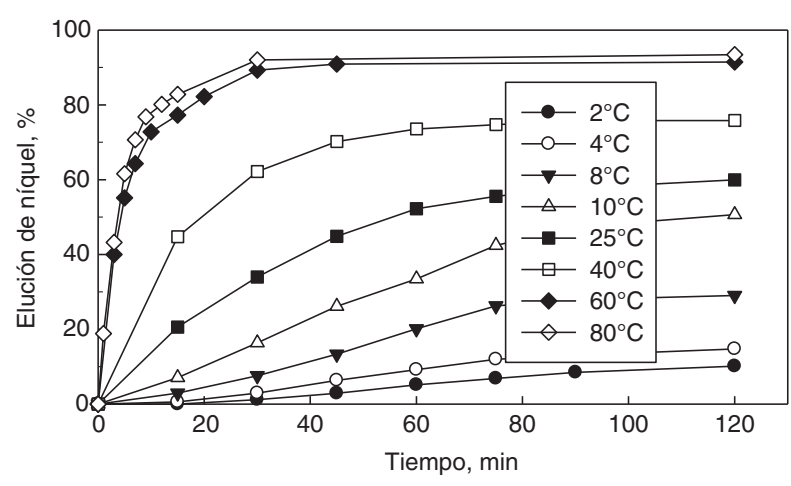

FIGURA 5. Efecto de la temperatura sobre la velocidad de elución de níquel. 
el efecto preponderante que tiene la temperatura sobre la velocidad de elución del níquel. Así, para una temperatura de $8{ }^{\circ} \mathrm{C}$ la elución de níquel es lenta y solamente se obtiene un $28 \%$ de elución en 120 minutos, mientras que a $80{ }^{\circ} \mathrm{C}$ se alcanza una elución sobre el 93\% al mismo tiempo.

También se puede observar que aun trabajando cerca del punto de solidificación del agua $\left(0^{\circ} \mathrm{C}\right)$, se puede obtener una significativa elución de níquel llegando a valores de $10 \%$ a 120 minutos.

Las grandes diferencias observadas en las velocidades de elución de níquel desde PDAB pueden ser debidas a los cambios de las constantes cinéticas que envuelven las velocidades de los procesos de elución.

\subsection{Efecto de la concentración de ácido sulfúrico}

Se estudió el efecto de la concentración de $\mathrm{H}_{2} \mathrm{SO}_{4}$ sobre la velocidad de elución de níquel. Los resultados son mostrados en la Fig. 6. Claramente se observa que con bajas concentraciones de ácido sulfúrico, la elución de níquel es muy lenta llegando a valores de elución de 4\% para una concentración de $0,01 \mathrm{~g} \mathrm{~L}^{-1} \mathrm{de}_{2} \mathrm{SO}_{4}$ y en un tiempo de 120 minutos. Sin embargo, incrementando la concentración de ácido hasta $2,5 \mathrm{~g} \mathrm{~L}^{-1}$, se incrementa notablemente la elución de níquel alcanzando un valor de $84 \%$ en el mismo tiempo experimental. Se debe hacer notar que utilizando una concentración de $0,005 \mathrm{~g} \mathrm{~L}^{-1}$ de $\mathrm{H}_{2} \mathrm{SO}_{4}$, no se observó elución alguna de níquel.

Por otro lado, se analizó la composición química superficial de níquel sobre el PDAB. Se realizó el análisis por DRX sobre las muestras cortadas con grafito; las partículas de PDAB fueron eliminadas desde los experimentos a tiempos de 30 y 120 minutos para una concentración de $\mathrm{H}_{2} \mathrm{SO}_{4}$ de $0,25 \mathrm{~g} \mathrm{~L}^{-1}$. Cada uno de los dos puntos fue analizado utilizando el sistema EDS. Las mediciones son presentadas en la Fig. 7. La Tabla 2 muestra un resumen de los resultados de la composición de níquel sobre la superficie de las esferas. Dicha tabla muestra que la composición promedio de níquel sobre la

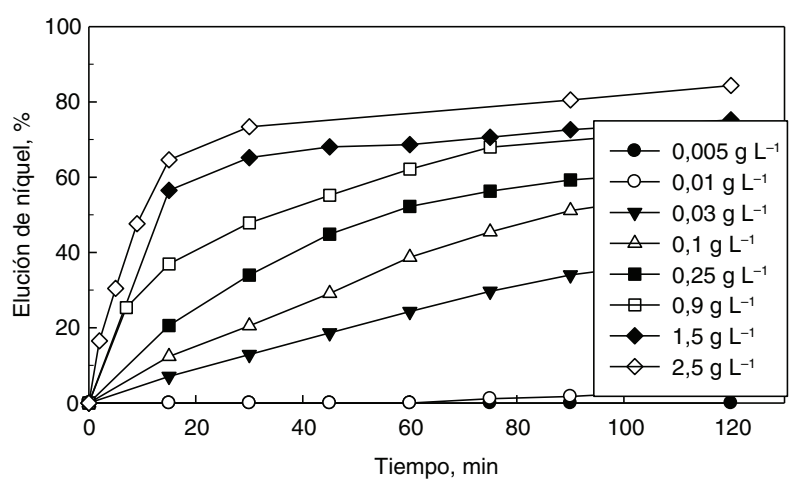

Figura 6. Efecto de la concentración de $\mathrm{H}_{2} \mathrm{SO}_{4}$ sobre la velocidad de elución de níquel. superficie de la partícula disminuye a medida que se incrementa el tiempo de permanencia del PDAB en la solución, el cual coincide con los resultados resumidos en la Fig. 6. La disminución alcanzó un valor de alrededor de $6,6 \%$ del contenido de níquel por incremento del tiempo experimental desde 0 a 30 minutos; un mayor decrecimiento de níquel en la superficie se obtiene a un tiempo de 120 minutos, observándose un decrecimiento de alrededor de $95 \%$.

\subsection{Efecto de distintos reactivos ácidos}

Para conocer el efecto que tuvieron distintos reactivos ácidos sobre la velocidad de elución de níquel, se realizaron pruebas con tres reactivos: $\mathrm{HNO}_{3}, \mathrm{HCl}$ y $\mathrm{HClO}_{4}$. Los resultados experimentales son mostrados en la Fig. 8, donde se puede apreciar que para los tres reactivos utilizados existió un efecto positivo en la elución de níquel. Así, utilizando $\mathrm{HNO}_{3}$ se obtiene un $65,8 \%$ de elución a los 120 minutos, mientras que utilizando $\mathrm{HCl}$ se llegó a una elución de $56,2 \%$ en el mismo tiempo. Las velocidades de elución, utilizando ambos reactivos $\left(\mathrm{HNO}_{3}\right.$ y $\left.\mathrm{HCl}\right)$, fueron similares a las curvas obtenidas con $\mathrm{H}_{2} \mathrm{SO}_{4}$. Sin embargo, al utilizar el reactivo $\mathrm{HClO}_{4}$, fue distinto el comportamiento de la curva de elución, el cual mostró una lentitud en la elución llegando a valores de 30,6\% de elución en un tiempo de 120 minutos. Por lo tanto, debido a la escasa bibliografía sobre la elución de níquel mediante el uso de estos reactivos ácidos, la utilización de otros reactivos ácidos hace necesario desarrollar estudios anexos para encontrar el efecto de éstos reactivos a bajas y mayores concentraciones.

\subsection{Estabilidad del alginato con distintos números de ciclos}

Se estudió la estabilidad de las esferas de alginato en función del número de ciclos. Para este punto se realizaron pruebas de extracción y elución a las mismas condiciones mencionadas anteriormente (Extracción: concentración inicial de $\mathrm{Ni}=17 \mathrm{mg} \mathrm{L}^{-1}$, un valor de $\mathrm{pH}$ de 5,0, masa de alginato utilizada de $80 \mathrm{mg}$, temperatura de la solución de $25^{\circ} \mathrm{C}$ y tiempo experimental de 360 minutos; Elución: concentración inicial de $\mathrm{H}_{2} \mathrm{SO}_{4}=0,25 \mathrm{~g} \mathrm{~L}^{-1}$, masa de alginato de $80 \mathrm{mg}$, temperatura de $60{ }^{\circ} \mathrm{C}$ y tiempo experimental de 60 minutos). Los ciclos llegaron hasta un número de cinco. Los resultados mostraron extracciones de hasta $75 \mathrm{mg} \mathrm{g}^{-1}$ y eluciones cercanas al 90\%, para cada una de los ciclos. Estos resultados pusieron de manifiesto que el alginato podría ser reutilizado manteniéndose las condiciones de trabajo. También se observó que el alginato mantuvo su estabilidad física, sin desprendimiento de su estructura ni tampoco destrucción de ella. Con estas pruebas se contempla realizar trabajos 

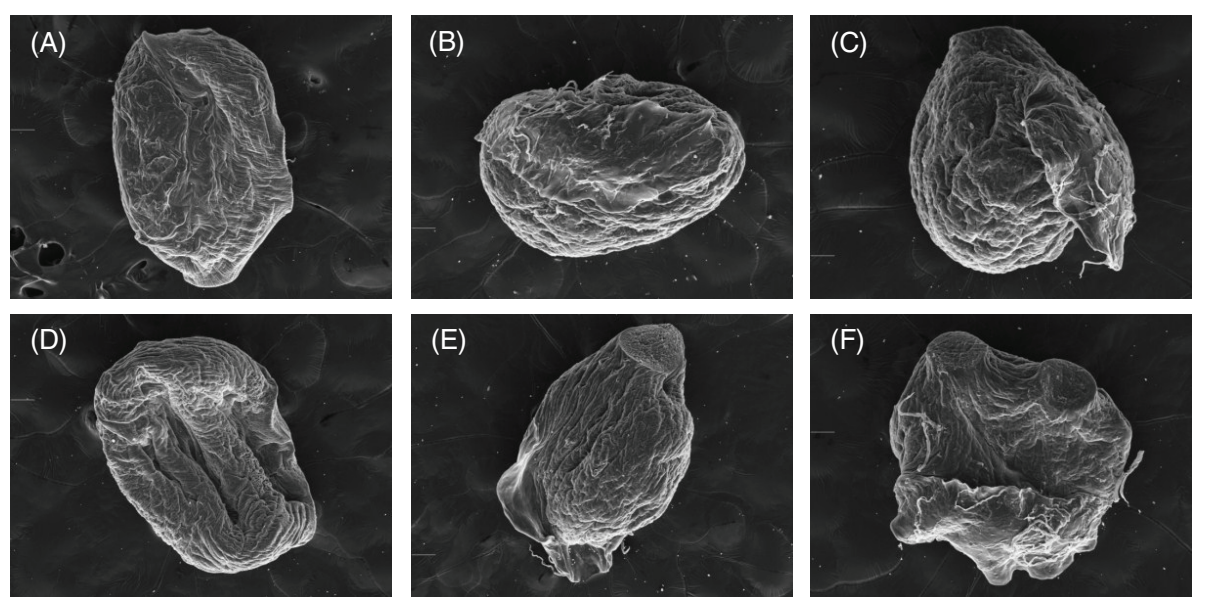

FIgURA 7. Micrografías BEI y mapeo del alginato; (A y D) Muestra original; (B y E) 30 minutos; (C y F) 120 minutos.

a futuro mediante un sistema en continuo para estudiar la cama de esferas, número de ciclos mayores y otras variables.

\subsection{Cinética de elución de níquel}

El proceso de elución de níquel fue notablemente afectado por la temperatura. Por lo tanto, se sugiere que el proceso de elución está controlado por la reacción química en la superficie. Asimismo, asumiendo el control de la reacción sobre la superficie, se aplicó a este proceso la ecuación de velocidad de núcleo recesivo para una partícula de PDAB de radio inicial $\mathrm{r}_{\mathrm{o}}$ para una concentración constante de reactantes. Tal ecuación fue descrita como (Shon y Wadsworth, 1979):

$$
1-(1-\alpha)^{1 / 3}=k_{\text {app }} t
$$

donde $\alpha$ es la fracción de níquel eluída, $t$ es el tiempo de elución, y $k_{a p p}$ es la constante cinética aparente dada por la siguiente expresión general:

$$
k_{\text {app }}=k_{1} C_{\mathrm{H}_{2} \mathrm{SO}_{4}}^{m}
$$

TABla 2. Concentración de Ni, expresada en $\%$, sobre la superficie del alginato por análisis de EDS

\begin{tabular}{lcccc}
\hline & & \multicolumn{3}{c}{ Concentración de Ni (\%) } \\
\cline { 3 - 5 } Partícula & Punto & Muestra original & 30 minutos & $\mathbf{1 2 0}$ minutos \\
\hline 1 & 1 & 10,52 & 3,35 & 0,51 \\
& 2 & 9,56 & 2,90 & 0,54 \\
& 3 & 9,29 & 2,98 & 0,48 \\
2 & 1 & 9,12 & 3,13 & 0,62 \\
& 2 & 9,67 & 2,31 & 0,61 \\
& 3 & 9,24 & 2,46 & 0,50 \\
\multirow{2}{*}{ Promedio } & & 9,53 & 2,98 & 0,52 \\
\hline
\end{tabular}

donde $k_{1}$ es la constante de velocidad lineal, $\mathrm{C}_{\mathrm{H}_{2} \mathrm{SO}_{4}}$ es la concentración en la solución de ácido sulfúrico y $m$ es el orden de reacción aparente

La Fig. 9 muestra un gráfico de $1-(1-\alpha)^{1 / 3}$ como una función del tiempo para los datos experimentales obtenidos desde la Fig. 5 en el rango de temperatura de 2 a $80^{\circ} \mathrm{C}$. Una aproximación similar fue

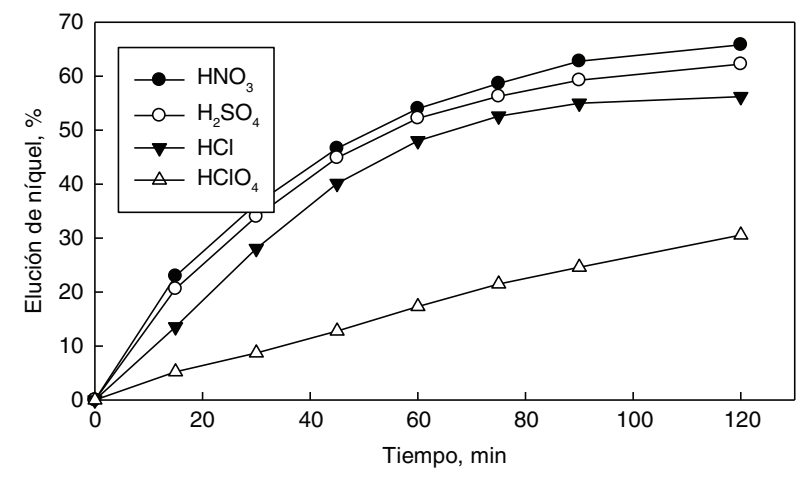

Figura 8. Efecto de diferentes reactivos ácidos sobre la elución de níquel.

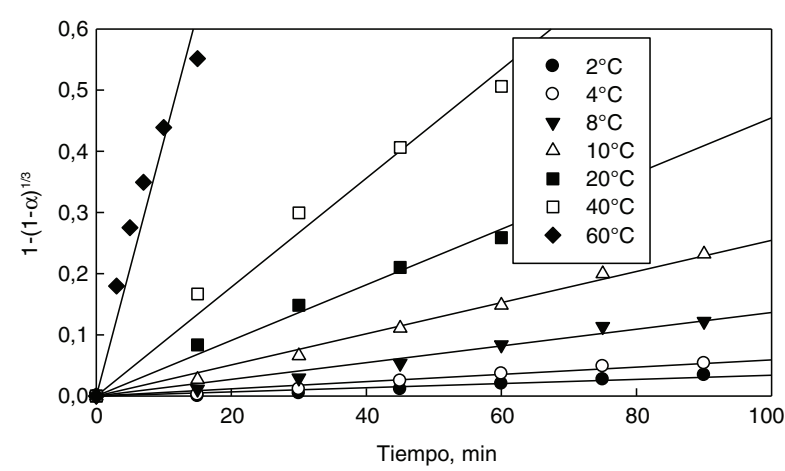

Figura 9. Cinética de elución de níquel en una solución de $\mathrm{H}_{2} \mathrm{SO}_{4}$ para las condiciones de elución de la Fig. 5. 
TABla 3. Constantes de velocidad para la elución de níquel

\begin{tabular}{lccc}
\hline $\mathbf{T}\left[{ }^{\circ} \mathbf{C}(\mathbf{K})\right]$ & $\mathbf{1 0 0 0 / T}(\mathbf{1} / \mathbf{K})$ & $\boldsymbol{k}_{\text {app }}$ & $-\ln \boldsymbol{k}_{\text {app }}$ \\
\hline $2(275)$ & 3,636 & 0,00034 & 7,9866 \\
$4(277)$ & 3,610 & 0,00059 & 7,4354 \\
$8(281)$ & 3,559 & 0,00136 & 6,6003 \\
$10(283)$ & 3,534 & 0,00254 & 5,9756 \\
$20(293)$ & 3,413 & 0,00455 & 5,3926 \\
$40(313)$ & 3,195 & 0,00860 & 4,7560 \\
$60(333)$ & 3,003 & 0,03989 & 3,2216 \\
\hline
\end{tabular}

utilizada para los resultados experimentales concernientes al efecto de la concentración de ácido sulfúrico. Se puede observar en esta figura un buen ajuste lineal de los datos cinéticos con coeficientes de regresión, $\mathbf{R}^{2}$, desde 0,96 a 0,99 para todo el rango de temperatura, indicando que se puede aplicar la Ec. (2). Los valores de las constantes cinéticas aparentes a varias temperaturas fueron obtenidas desde las pendientes de las líneas rectas y son mostradas en la Tabla 3.

Por lo demás, el orden de reacción $m$ fue calculado desde los datos cinéticos sobre el efecto de la

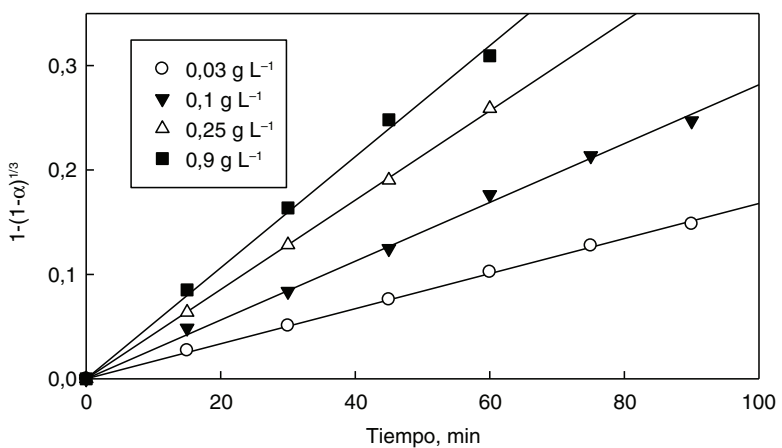

Figura 10. Cinética de elución a una temperatura de $25^{\circ} \mathrm{C}$ a diferentes concentraciones de $\mathrm{H}_{2} \mathrm{SO}_{4}$ para condiciones de elución de la Fig. 6.

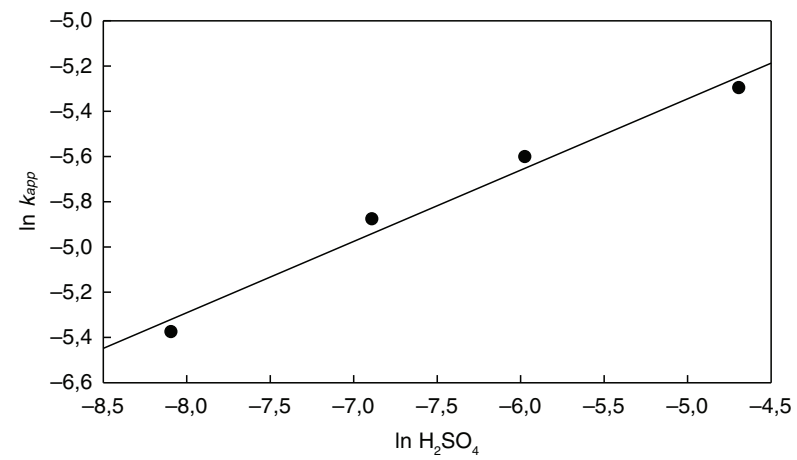

FIgURa 11. Orden de reacción con respecto a la concentración de $\mathrm{H}_{2} \mathrm{SO}_{4}$. concentración de ácido sulfúrico. La Fig. 10 muestra los datos experimentales para varias concentraciones de $\mathrm{H}_{2} \mathrm{SO}_{4}$ calculados de acuerdo a la Ec. (2), y los valores de $k_{a p p}$ fueron utilizados para representar un gráfico de $\ln k_{a p p}$ vs $\ln C_{\mathrm{H}_{2} \mathrm{SO}_{4}}$ como se muestra en la Fig. 11. Los datos de concentración de $\mathrm{H}_{2} \mathrm{SO}_{4}$ fueron transformados a concentración molar (M). En la Fig. 11 se observa una relación lineal con un $\mathrm{R}^{2}$ igual a 0,92 y una pendiente que indica que la reacción es de orden 0,33 con respecto a la concentración de ácido sulfúrico.

Seguidamente se calcularon las constantes de velocidad intrínseca $k_{1}$ para el rango de temperaturas estudiado mediante el uso de los valores de las constantes cinéticas aparentes obtenidas desde la Fig. 5 y el valor de $m$ igual a 0,33 . La Tabla 4 muestra ambas constantes de velocidad aparente e intrínseca para las temperaturas estudiadas. A continuación, los valores calculados de las constantes intrínsecas $k_{1}$ fueron utilizados para obtener el gráfico de Arrhenius mostrado en la Fig. 12. Esta figura muestra un buen ajuste lineal $\left(\mathrm{R}^{2}>0,92\right)$ de las constantes cinéticas intrínsecas de cada temperatura. La energía de activación calculada fue de $54,5 \mathrm{~kJ} \mathrm{~mol}^{-1}$ para el rango de temperatura entre $2 \mathrm{y}$ $60{ }^{\circ} \mathrm{C}$, el cual es un típico valor para una reacción controlada por reacción química en la superficie.

TABLA 4. Constantes de velocidad para la elución de níquel en el rango de temperaturas de 2 a $60{ }^{\circ} \mathrm{C}$ para una concentración de ácido sulfúrico de $2,55 \times 10^{-3} \mathrm{M}$

\begin{tabular}{lcc}
\hline $\mathbf{T}\left({ }^{\circ} \mathbf{C}\right)$ & $\boldsymbol{k}_{a p p}\left(\mathbf{m i n}^{-\mathbf{1}}\right)$ & $\boldsymbol{k}_{\boldsymbol{l}}\left(\mathbf{m i n}^{-\mathbf{1}}\right)$ \\
\hline 2 & 0,00034 & 0,00244 \\
4 & 0,00059 & 0,00423 \\
8 & 0,00136 & 0,00976 \\
10 & 0,00254 & 0,01822 \\
20 & 0,00455 & 0,03264 \\
40 & 0,00860 & 0,06169 \\
60 & 0,03989 & 0,28619 \\
\hline
\end{tabular}

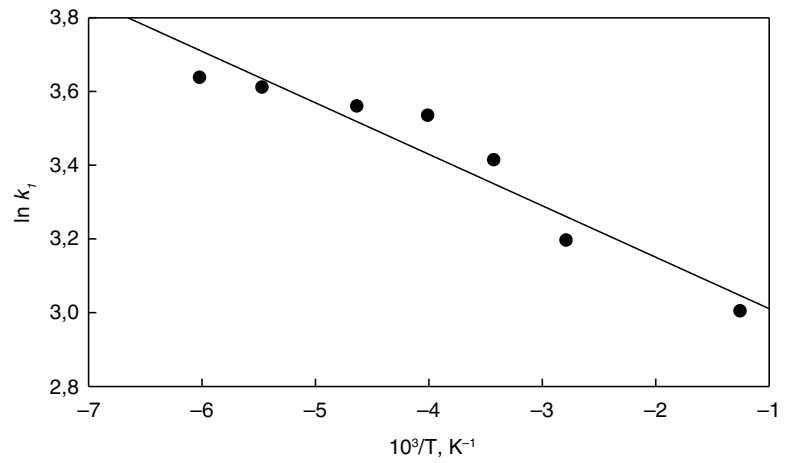

Figura 12. Gráfico de Arrhenius para la elución de níquel en una solución de ácido sulfúrico. 
Así, la cinética de elución de iones de níquel en un ambiente de ácido sulfúrico puede ser representada por la siguiente ecuación:

$1-(1-\alpha)^{1 / 3}=3,66 \times 10^{7} \cdot\left[\mathrm{H}_{2} \mathrm{SO}_{4}\right]^{0.33} \cdot \exp \left(-\frac{54,500}{\mathrm{RT}}\right) t$

donde $\mathrm{R}$ es igual a $8,314 \mathrm{~J} \mathrm{~mol}^{-1} \mathrm{~K}^{-1},\left[\mathrm{H}_{2} \mathrm{SO}_{4}\right]$ está en concentración molar, $t$ es en minutos $\mathrm{y}$ $k_{1}=3,66 \times 10^{7} \mathrm{M}^{-0,33} \mathrm{~min}^{-1}$.

\section{CONCLUSIONES}

De los resultados de esta investigación, se pueden enumerar las siguientes conclusiones:

- La elución de níquel desde esferas de alginato se produce por un mecanismo de intercambio iónico entre protones e iones de níquel.

- Al incrementar la velocidad de agitación de la solución se produce un aumento de la elución de iones de níquel debido a que la transferencia de masa no juega un rol importante.

- El aumento de la temperatura y concentración de ácido sulfúrico permite incrementar la elución de níquel hasta llegar a valores de 93\% de elución a una temperatura de $80^{\circ} \mathrm{C}$ y concentración de $\mathrm{H}_{2} \mathrm{SO}_{4}$ de $0,25 \mathrm{~g} \mathrm{~L}^{-1}$.

- La cinética de elución de níquel fue analizada utilizando un modelo $1-(1-\alpha)^{1 / 3}=k_{a p p} \mathrm{t}$, que permitió la determinación de la dependencia de la temperatura y concentración del ácido sulfúrico sobre la velocidad de elución.

- Se encontró que la reacción de intercambio iónico es controlada por la reacción química en la superficie y es de orden 0,33 con respecto a la concentración de ácido sulfúrico y la energía de activación fue determinada como $54,5 \mathrm{~kJ} \mathrm{~mol}^{-1}$ para el rango de temperatura entre 2 y $60{ }^{\circ} \mathrm{C}$.

\section{REFERENCIAS}

Anoop, K., Sreejalekshmi, K.G., Baiju, R.S. (2011). Nickel(II) adsorption onto biomass based activated carbon obtained from sugarcane bagasse pith. Bioresource Technol. 102 (22), 10239-10247. http://dx.doi.org/10.1016/j.biortech. 2011.08.069.

Aracena, A., Guajardo, N., Ibáñez, J.P., Jerez, O., Carlesi, C. (2015). Uptake of nickel ions from aqueous solutions using protonated dry alginate beads. Can. Metall. Quart. 54 (1), 58-65, http://dx.doi.org/10.1179/1879139514Y.0000000152.

Belova, D.A., Lakshtanov, L.Z., Carneiro, J.F., Stipp, S.L.S. (2014). Nickel adsorption on chalk and calcite. J. Contam. Hydrol. 170, 1-9. http://dx.doi.org/10.1016/j.jconhyd.2014. 09.007 .

Ibáñez, J.P., Umetsu, Y. (2002). Potential of protonated alginate beads for heavy metals uptake. Hydrometallurgy 64 (2), 89-99. http://dx.doi.org/10.1016/S0304-386X(02)00012-9.

Ibáñez, J.P., Umetsu, Y. (2008). Uptake of $\mathrm{Cd}^{2+}$ from aqueous solutions using protonated dry alginate beads. Can. Metall. Quart. 47 (1), 45-50. http://dx.doi.org/10.1179/cmq.2008. 47.1.45.

Ibáñez, J.P., Aracena, A. (2014). Uptake of $\mathrm{Zn}^{2+}$ from dilute aqueous solutions using protonated dry alginate beads. Can Metall. Quart. 53 (1), 82-87. http://dx.doi.org/10.1179/1879 139513Y.0000000104

Malanis, S., Katsou, E. (2013). A review of zinc and nickel adsorption on natural and modified zeolite, bentonite and vermiculite: Examination of process parameters, kinetics and isotherms. J. Hazard. Mater. 252-253, 428-461. http:// dx.doi.org/10.1016/j.jhazmat.2013.03.024.

O’Connell, D.W., Birkinshaw, C., O’Dwyer, T.F. (2008). Heavy metal adsorbents prepared from the modification of cellulose: A review. Bioresource Technol. 99 (15), 6709-6724. http:// dx.doi.org/10.1016/j.biortech.2008.01.036.

Roine, A. (1999). HSC Chemistry 6.0 Sofware, OutoKumpu Research Py, Pori, Finlandia.

Shon, H.Y., Wadsworth, M.E. (1979). Rate processes of extractive metallurgy, Plenum Press, New York, USA

Vocciante, M., Trofa, M., Rodríguez, P., Giraldo, L., D’Auria, T., Moreno, J.C., Erto, A. (2014). A rigorous procedure for the design of adsorption units for the removal of cadmium and nickel from process wastewaters. J. Clean. Prod. 77, 35-46. http://dx.doi.org/10.1016/j.jclepro.2013.12.001.

Xia, L., Hu, Y., Zhang, B. (2014). Kinetics and equilibrium adsorption of copper(II) and nickel(II) ions from aqueous solution using sawdust xanthate modified with ethanediamine. T. Nonferr. Metal. Soc. China 24 (3), 868-875. http://dx.doi. org/10.1016/S1003-6326(14)63137-X. 